\title{
Analysis of Urban Roads Traffic Impact Based On IoT
}

\author{
SudhaRajesh ${ }^{1}$, Mahalakshmi $^{2}$, Meena $^{3}$ \\ Assistant Professor ${ }^{1}$, Department of Computer Science and Engineering ${ }^{1,2,3}$ \\ Jeppiaar SRR Engineering college ${ }^{1,2,3}$ \\ Email:maha23oct97@gmail.com ${ }^{2}$,meenasrinivasan7@gmail.com ${ }^{3}$
}

\begin{abstract}
Fast moving of vehicles and over congestion of vehicles on roads, highways leads to major accidents nowadays. Collision of vehicles due to unstable condition of drivers and violation of traffic rules are the other reasons of accidents. Generally, fixed sensors on motorized vehicles help the users to get alert notifications about the traffic and it is very useful for ambulance. But, unfortunately it does not prevents accident. To overcome this issue an alternative solution considering the halting of the vehicles engine automatically that violate the traffic signal. This solution gives a drastic change in reducing accidents. Here an IoT cloud system is used for identification of vehicles that violate the rules. Instant deduction of fine amount would make them not to breach the rules. Various demonstrations prove that our system avoids major risk accidents occurring on highways and urban roads.
\end{abstract}

Index Terms- congestion, collision, halting, deduction

\section{INTRODUCTION}

Traffic impact analysis can have significant adverse social, economic and environmental impacts within densely populated urban areas. A traffic incident is one of the major factors contributing to traffic congestion. Traffic incident refers to any event that degrades safety and slows traffic, including disabled vehicles, accidents, debris on the roadway and hazardous material spills; it basically reduces the roadway capacity. On a nearly saturated road, a small reduction in its capacity can lead to a dramatic increase in traffic congestion. Most major roads in cities are operating at/or above capacity during peak hours resulting in an increasing potential for traffic incidents and significant delay to the travellers. There is a need to address the incident problem in order to avoid the resulting accidents, which also may result in one or more of the following (i) wasting time of motorists and passengers (ii) inability to forecast travel time accurately (iii) wasted fuel, increased air pollution and carbon dioxide emissions owing to increased idling, acceleration and braking (iv)damage that occur naturally and inevitably as a result of idling in traffic and frequent acceleration and braking, leading to more frequent repairs and replacements (v) stressed and frustrated motorists, encouraging road rage and reduced health of motorists (vi) blocked traffic interfering with the passage of $\mathbf{1}$. emergency vehicles, and (vii) increased vehicle operating costs due to more fuel usage. Traffic impact analysis (TIA) deals with the general study of the extent or degree to which the preferable land use developments, and the traffic are expected to generate, will affect the adjacent or surrounding transportation system. The intiative of those studies is often to determine which lay of roadway capacity improvements might be needed to support the anticipated demand and/or to mitigate the adverse effects of the increase in volume.In most cases, vehicular accidents are caused by avoidable human errors and improper driving practices. With advances in recent sensing technologies, autonomous vehicles and connected cars are becoming more and more practicable. A distributed system sharing sensor data coming from vehicles can reduce the accidents by the use of direct or indirect vehicle to vehicle $(\mathrm{V} 2 \mathrm{~V})$, vehicle to infrastructure (V2I) and infrastructure to vehicle (I2V) interactions. Sensor technology connected with cars allows drivers to improve their driving experience. This enables warnings and precautions from a network of road side units,functioning as stationary way points [1]. Such information is particularly beneficial for drivers in remote areas where roads cannot be equipped with traffic sensors.Incident includes vehicle breakdowns, debris on the road, roadside distractions, spilled loads and accidents. With a good knowledge of incident-induced congestion,transportation agencies will be able to select more effective counteract against roadway traffic congestion. Special attention should be paid to travel delays caused by incident related congestion which may be cost-effectively alleviated through traffic management, control and incident response. To mitigate Incident Induced Delay (IID), a better understanding of incident impacts on traffic and traffic evolution during an incident is indispensable.

\section{BACKGROUND}

In this section, we'll offer background info necessary to know our motivations and therefore the ideas behind the model projected during this paper.

\subsection{Internet of things (IoT)}

IoT systems that allow users to achieve deeper analysis, automation and integration within a system. They improve the reach of suggested areas 


\section{International Journal of Research in Advent Technology, Vol.7, No.2, February 2019 E-ISSN: 2321-9637 \\ Available online at $w w w . i j r a t . o r g$}

and their accuracy. IoT utilizes existing and rising technology for sensing, networking, and robotics. IoT exploits recent advances in code, falling hardware prices, and modern attitudes towards technology. Its new and advanced components bring major changes within the delivery of merchandise, goods, and services; and the social, economic, and political impact of those changes. The Internet of things refers to a sort of network to connect one thing with internet supported stipulated protocols through information sensing equipments to conduct information exchange and communications in order to achieve smart recognitions, positioning, tracing, monitoring, and administration. In this paper we briefly discussed about what IoT is, how IoT enables different technologies, about its architecture, characteristics \& applications, IoT functional view \& what are the future challenges for IoT. IoT has recently become additional relevant to the sensible world for the most part due to the expansion of mobile devices, embedded and ubiquitous communication, cloud computing and data analytics.

\subsection{Technologies}

Enabling technologies for the Internet of Things are thought-about in and might be sorted into many categories: (1) technologies that change "things" to accumulate discourse info, (2) technologies to improve security and privacy. The first 2 classes will be together understood as useful building blocks needed building "intelligence" into "things", that square measure so the options that differentiate the IoT from the same old net.The other categories are not a functional but rather a de-facto requirement, without which the penetration of the IoT would be severely reduced.The Internet of Things is not a single technology, but it is a mixture of different hardware \& software technology .The Internet of Things provides solutions supported the combination of knowledge technology, which refers to hardware and software used to store, retrieve, and process data and communications technology which includes electronic systems used for communication between people or teams.

\subsection{Key Features}

The most vital options of IoT embody AI,property,Sensors, active engagement, and small device use .A brief review of those options is given below: AI - IoT basically makes nearly something "smart", meaning it enhances every aspect of life with the power of data collection, artificial intelligence algorithms, and networks. This

can mean one thing as straight forward as enhancing passive network of devices into a lively system capable of real-world fuse. Active Engagement plenty of today's interaction with connected technology happens through passive engagement. IoT introduces a replacement paradigm for active content, product, or service engagement. Small Devices - Devices, as predicted, became smaller, cheaper, and tons of powerful over time. IoT exploits purposeful tiny devices to deliver its exactness, scalability, and versatility.

\subsection{IoT Software}

IoT code addresses its main areas of networks and action through platforms, embedded systems, partner systems, and middleware. These individual and master applications square measure chargeable for knowledge assortment, device integration, realtime analytics, and application and process extension within the IoT network. They exploit integration with essential business systems (e.g., ordering systems, robotics, scheduling, and more) in the execution of related tasks.

\subsection{Data assortment}

This software collection manages anticipate, analysis, light data refining, light data shield, and gathering of data. It uses sure protocols to assist sensors in connecting with period, machine-tomachine networks. Then it collects knowledge from multiple devices and distributes it in accordance with settings. It additionally works in reverse by distributing knowledge over devices. The system eventually transmits all collected knowledge to a central server.

\subsection{Device Integration}

Software system aiding crunch all the system devices to make the body of the IoT system. It ensures the desired cooperation and steady networking between devices. These applications units measure the shaping code technology of the IoT network as a result of while not them, it is not an IoT system. They manage the assorted applications, protocols, and limitations of each device to allow communication.

\subsection{Real-Time Analytics}

These applications take knowledge or input from numerous devices and convert it into viable actions or clear patterns for human analysis. They analyze info supported numerous settings and styles so as to perform automation-related tasks or offer the info needed by business.

\subsection{Application and Process Extension}

These applications extend the reach of existing systems and code to permit a wider, more effective system. They integrate predefined devices for specific functions like permitting sure mobile devices or engineering instruments access. It supports improved productivity and additional correct knowledge collect.

\subsection{Traffic Impact Analysis}

Total car traffic control is the primary objective of any traffic authority. Considering an urban context, 
the idea of traffic control raises many application scenarios. Traffic regulation has a subsequent positive impact in terms of environment, accident prevention, speeding up vehicular circulation, optimizing public transportation, and social aspects. Traffic congestion is a severe problem on highways. According to a study, its impact will increase even up to $2 \%$ every year. Since building new infrastructure is no longer an appropriate option in most countries, there are many approaches towards a more effective road usage and a more intelligent way of increasing the capacity of the road network. Examples of advanced traffic management systems area unit are intelligent speed limits, monitoring traffic violation, accommodative ramp metering, or dynamic routing. These examples are based on a centralized traffic management, which controls the operation and the response according to a giventraffic situation.

\section{RELATED WORK}

There are several existing works within the domain of IoT. In this section, we are going to discuss the foremost current notable works.

Antonio Celesti, Antonino Galletta, Lorenzo Carnevale undertook a literary and experimental work on An IoT Cloud System for Traffic Monitoring and Vehicular Accidents Prevention Based on Mobile Sensor Data Processing [1].

The abrupt traffic delay particularly in quick scrolling roads and highways characterised by a scarce visibility is one in every of the main causes of accidents among motor vehicles. It will be caused by alternative accidents, work-in-progress on roads, excessive motorized vehicles especially at peak times and so on. Typically, mobile traffic sensors directly installed in private and/or public transportation and volunteer vehicles. In this scenario a fast real time processing of big traffic data is fundamental to prevent accidents. In specific, we tend to discuss an IoT Cloud system for traffic watching and alert notification supported OpenGTS and MongoDB. Our IoT Cloud system, besides for private drivers, it is very useful for drivers of critical rescue vehicles such as ambulances. Experiments prove that our system provides acceptable response times that allows drivers to receive alert messages in useful time so as to avoid the risk of possible accidents.

In another existing work, Iftikhar Ahmad, RafidahMd Noor, Ihsan Ali, Muhammad Imran and AthanasiosVasilakos on Characterizing the role of vehicular cloud computing [3].

Vehicular cloud computing is pictured to deliver services that give traffic safety and potency to vehicles.Vehicular cloud computing as nice potential to vary the up to date transport communication paradigm. Explicitly, the underutilized resources of vehicles will be shared with alternative vehicles to manage traffic throughout congestion. These resources embody however don't seem to be restricted to storage, computing power, and Internet connectivity. This study reviews current traffic management systems to research the role and significance of transport cloud computing in road traffic management. First, an abstraction of the transport cloud infrastructure in an urban situation is conferred to explore the transport cloud computing method.A taxonomy of transport clouds that defines the cloud formation, integration types, and services is presented. A taxonomy of transport cloud services is additionally provided to explore article sorts concerned positions among the transport cloud. A comparison of latest traffic management systems is performed in terms of parameters, such as vehicular adhoc network infrastructure, Internet dependency,cloud management, scalability, traffic flow control, and emerging services. Potential future challenges and rising technologies, such as the Internet of vehicles and its incorporation in traffic congestion control, are also discussed. Vehicular cloud computing is envisioned to have a substantial role within the development of good traffic management solutions and in rising net of vehicles. Aimé Lay-Ekuakille ,Patrizia Vergallo, Davide Saracino, and AmerigoTrotta undertook a literary and experimental work on Optimizing and Post Processing of a Smart Beamformer for Obstacle Retrieval [4].

Beamforming is one in every of the foremost grabbing techniques accustomed understand distance systems so as to notice timely, widespread obstacles. If correctly associated to DOA (Difference of Arrival), it can allow the description of obstacle shape. Distance move, for mobile and fixed systems, namely cars, vehicles, vessels and airplanes, that is a key issue for demands of nowadays. Distance between cars and from obstacles will be established and measured victimization optical device and ultrasound.Cloudy and foggy conditions area unit important necessities for testing distance move facilities.If based on acoustic waves, they can be easily integrated by sophisticated on-board software in order to perform new features. This analysis presents attention grabbing aspects of defining new necessities for an acoustic scanning capable of reconstructing fixed obstacle options by targeting them employing a special array of sensors. The term "acoustic scanning" is meant here as a facet of mensuration and replic relating to spatial locations of the obstacle, that's spatial shaping. The paper illustrates an experimental system first from which it is possible to derive parameters for setting spatial shaping of scenarios and after a clear identification of DOAs.

\section{PROPOSED SYSTEM}

All metropolitan cities face tie up issues particularly in the downtown areas. The paradigm of Internet of 


\section{Available online at www.ijrat.org}

Things (IoT) can play an important role in realization of smart cities. IoT based traffic management provide solutions for Traffic violations which are been identified and centrally monitored or controlled through Internet. The vehicle that doesn't obey the traffic signals are provided with unique identifiers. However this can be used in any Metropolitan city without the loss of generality. In this system, when vehicle crosses the signal if it is red a camera which is placed at the traffic signal captures the vehicle number[2].The vehicle number is sent to cloud through node mcu and retrieves back the details of a person and vehicle. Using Arduino (i.e) microcontroller we can stop the vehicle engine. The user has to show Radio frequency identification(RFID) tag to the nearby RFID receiver.If the user has paid the fine amount, the arduino starts vehicle engine. This is clarified in the below Figure 1. The status of the traffic signal is been checked continuously. The vehicle is monitored by using camera.

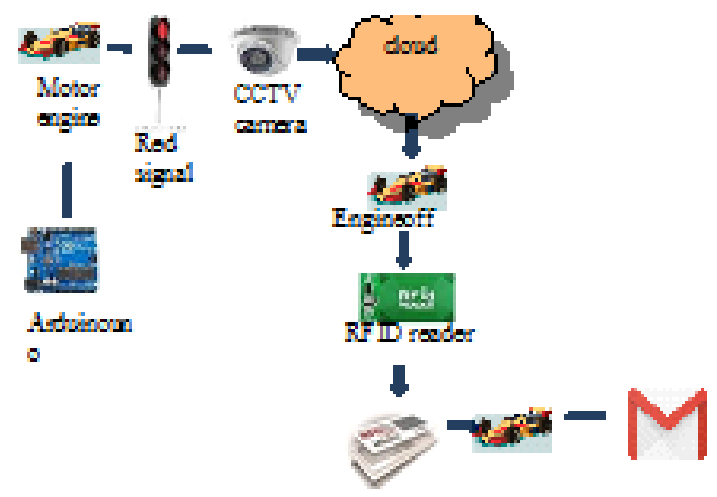

Figure 1: System Architecture

\subsection{Monitoring traffic signal status}

Whenever a vehicle is seen crossing the signal when it is in red the camera captures the number plate immediately. The vehicle is connected to arduino and node mcu. The node mcu acts as an intermediate between the arduino and cloud.After the camera capturing the number plate image it sends the image to the cloud server immediately. These concepts are depicted in the below Figure 2.

\section{Figure 2: Traffic signal status}

S.U.R.F or Speeded Up Robust Features is a patented algorithm used mostly in computer vision tasks and tied to object detection purposes. SURF fall in the category of feature descriptors by extracting key points from different regions of a given image and thus is very useful in finding similarity between images.In SIFT Lower approximated Laplacian of mathematician with distinction for locating scale-space. SURF goes a bit more and approximates LoG with Box Filter. Below Figure 3 shows an indication of such associate degree approximation. One huge advantage of this approximation is that, convolution with box filter can be easily calculated with the help of integral images and it is worn out parallel for various scales. Also the SURF admit determinant of the Wellington boot matrix for each scale and placement.
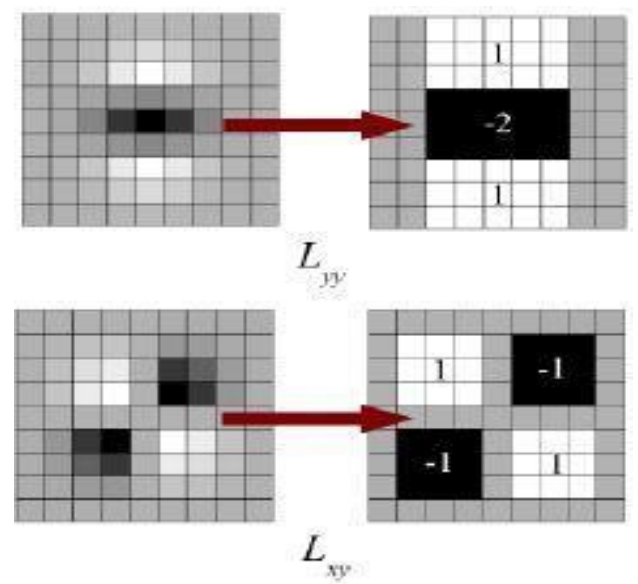

Figure 3: Surf Algorithm

\subsection{Vehicle identification}

Surf algorithm is used to extract specific vehicle number plate image which is implemented by MATLAB. Image processing algorithm is comparing CCTV image with data base image. The MATLAB is connected to the controller and then the controller is connected with Node MCU. These intellections are illuminated in below Figure 4.

\subsection{Halting of vehicle engine}

Node MCU which sends an URL to the cloud and gets the details of the vehicle. Node MCU sends a stop command to the controller. The respective vehicle engine is stopped by Arduino(controller).

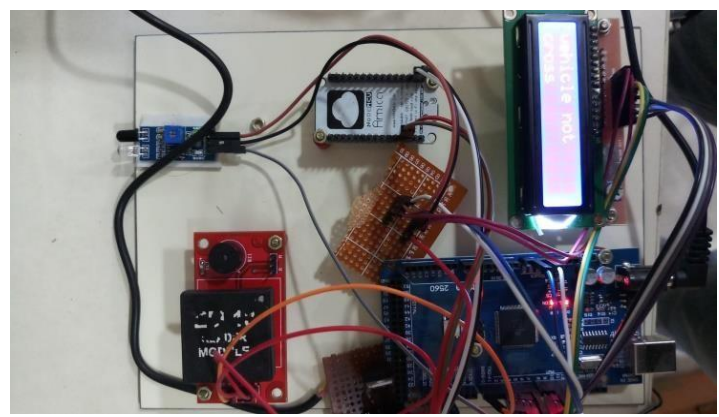

Figure 4: Vehicle identification

\subsection{Deduction of fine Amount}

The user has to show RFID tag to the nearby RFID 


\section{Available online at www.ijrat.org}

receiver which is placed anywhere near the signal

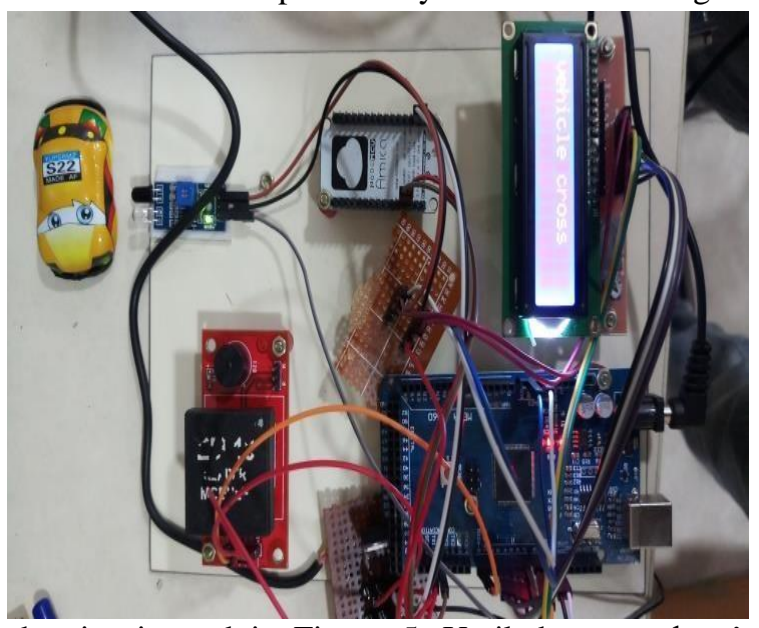

that is pictured in Figure 5. Until the user show's RFID the vehicle would be idle. If the user has paid the fine amount, the Node MCU sends a start command to the controller. Then the arduino starts vehicle engine. Finally, a mail will be sent to the user's mail id, such that it contains the deduction amount / penalty for traffic violation.

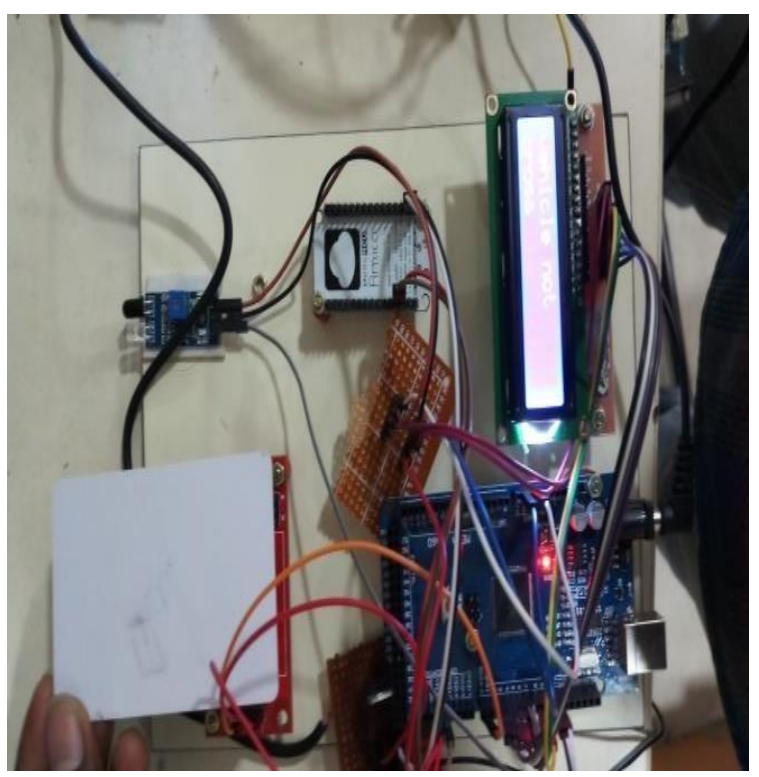

Figure 5: RFID Tag

\section{RESULTS}

The results of traffic impact analysis are discussed below:

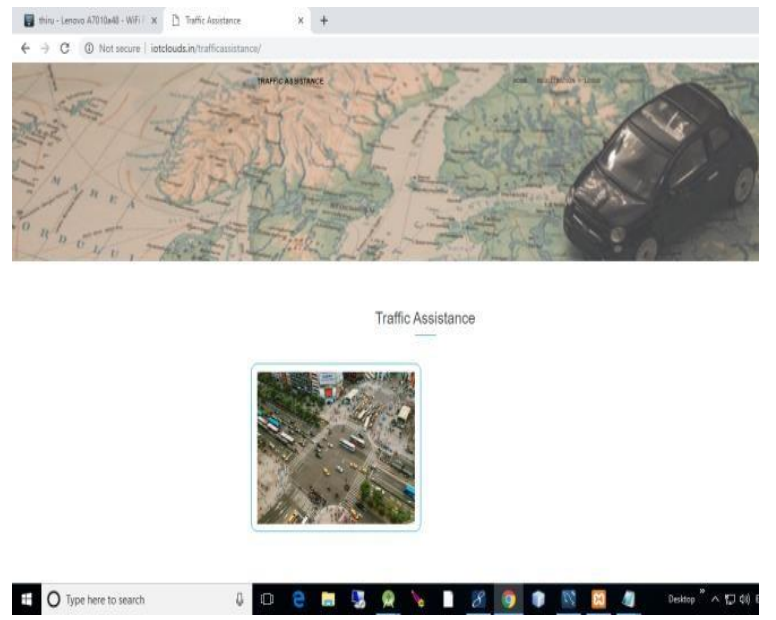

Figure 6: Traffic Assitance

The above figure 6 illustrates that traffic is being monitored continuously and the violation is detected.

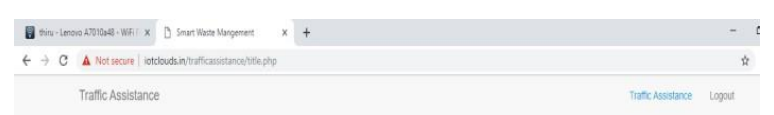

Traffic Assistance

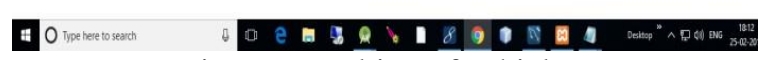

Figure 7: Halting of vehicle

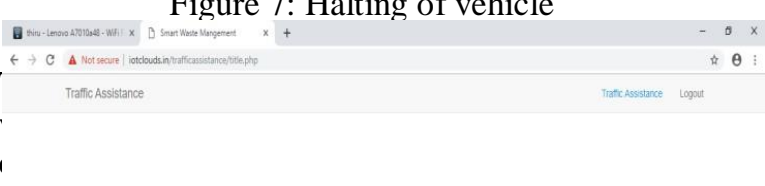

Traffic Assistance

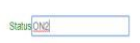

A 0

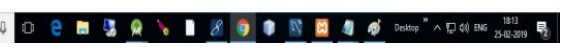

Figure 8: Activating vehicle engine

The above Figure 8 exemplifies that after paying the penalty for violation, the vehicle is started. The overhead Figure 9 represents that an e-mail would be 
sent to User's mobile after he has paid the fine amount.

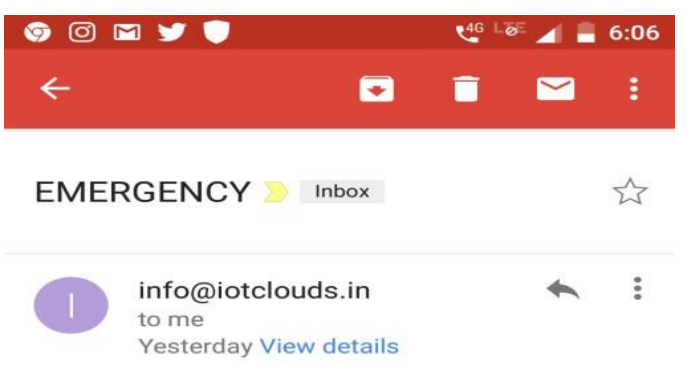

\section{Fine amount has Been Paid Your Vechile Will Be Starting In Few Minutes}

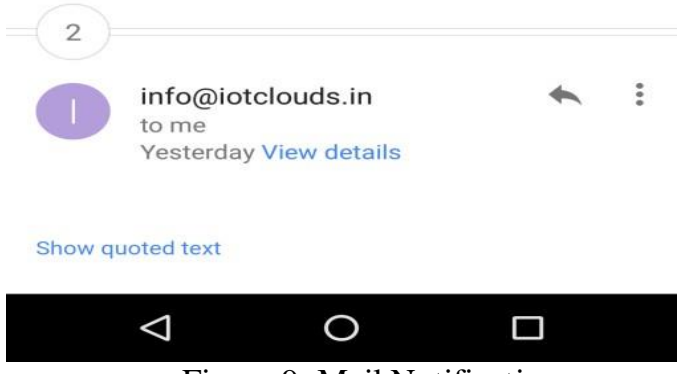

Figure 9: Mail Notification

\section{CONCLUSION \& FUTURE INCLUSIONS}

In this paper, we have discussed the problems faced by existing traffic violaton techniques. In response to this we have proposed a better way to prevent violation. The betterment of traffic condition in the towns are dependent on the newest ways of traffic mainframe and supervise. Advanced traffic signal controllers and control system contribute to the improvement of the urban traffic problem .Good quality social benefit has been made through the application of the intelligent traffic controller in practice, and the application result shows that the intelligent traffic signal controller will improve.Traffic based project thus helps to reduce the accidents occurring due to some persons irresponsible behaviour to cross the road.It is important that appropriate measures are taken to reduce the effect of incidents. Traffic control measures should therefore be taken to ensure efficient utilisation of the remaining road capacity during an incident. The traffic police department should improve vehicle inspection to reduce the number of accidents that cause congestion on roads. A penalty should be introduced for irresponsible behaviour such as running out of fuel that also contribute to congestion. The penalty for reckless driving which contributes to accidents should be increased to ensure safe driving and smooth flow of traffic. The road network in the city need to be improved to have alternative routes for vehicles once an incident has occurred. The traffic police officers should take accident measurements quickly and clear the incident instead of allowing negotiations between the two parties involved in an accident to take place at the scene.

\section{REFERENCES:}

[1] Antonio Celesti, AntoninoGalletta, Lorenzo Carnevale, Maria Fazio, Aime Lay-Ekuakille, and Massimo Villari, "An IoT Cloud System for Traffic Monitoring and Vehicular Accidents Prevention Based on Mobile Sensor Data Processing," IEEE sensors journal, vol. 18, no. 12, June 15, 2018.

[2] Ling $\mathrm{Hu}$, Qiang $\mathrm{Ni}$, "IoT-Driven Automated Object Detection Algorithm for Urban Surveillance Systems in Smart Cities", IEEE Internet of Things Journal, Volume:5 , Issue:2, pp.747-754, 2018.

[3] Iftikhar Ahmad, RafidahMd Noor, Ihsan Ali,Muhammad Imran and AthanasiosVasilakos, "Characterizing the role of vehicular cloud computing in road traffic management", International Journal of Distributed Sensor Networks, Volume:13, no:5 , pp.123-131, Dec 2017.

[4] Aimé Lay-Ekuakille , PatriziaVergallo, DavideSaracino, and AmerigoTrotta, "Optimizing and Post Processing of a Smart Beamformer for Obstacle Retrieval", IEEE Sensors Journal, Volume:12 , Issue:5 , pp.1294$1299,2012$. 